\title{
Low-temperature dielectric measurements of confined water in porous granites
}

\author{
Hugo Gonçalves Silva ${ }^{1}$, Mouhaydine Tlemçani ${ }^{2}$, Jorge Humberto Monteiro ${ }^{3}$, Manuel Pedro \\ Graça $^{3}$, Patricia Moita ${ }^{4}$, António Pinho ${ }^{5}$, Mourad Bezzeghoud ${ }^{2}$, Sushil Kumar Mendiratta ${ }^{3}$, and \\ Rui Namorado Rosa ${ }^{2}$
}

\begin{abstract}
The dielectric behavior of rocks affected by the known phase transition of supercooled water is the main problem we analyzed. Three different granitic rocks were used to perform dielectric measurements in the frequency range from $100 \mathrm{~Hz}$ to $1 \mathrm{MHz}$ and temperatures $100-350 \mathrm{~K}$. Thin cylindrical samples were prepared, and circular electrodes were established using silver conductive paint. A clear change in the dielectric measurements appears at $T \sim 220 \mathrm{~K}$ for one of the samples. This coincides with the known phase transition of supercooled water. Tightly bounded water confined in the pores of the rock do
\end{abstract}

not crystallize at $273 \mathrm{~K}$, but form a metastable liquid down to 200-220 K maintaining water polarization. Below this temperature, water molecules solidify and polarizability decreases. The rock presenting the most sizable change has a very low specific surface area of $\sim 0.09 \mathrm{~m}^{2} \mathrm{~g}^{-1}$, has connected porosity of $\sim 1.10 \%$, and has the smallest degree of alteration. In addition, geochemical analyzes reveal a low percentage of hydration water in its structure confirming the role of pore water in this change. A comparison between water-saturated, oven-dried, and vacuum-dried samples was done. Finally, a logarithmic dependency of the critical temperature for the supercooled water phase transition with the measuring frequency was found.

\section{INTRODUCTION}

Initial work on the dielectric behavior of rocks affected by pore water goes back to the 1960s (Howell and Licastro, 1961). The authors study different water unsaturated and dry rocks and minerals, and they find an increase in the dielectric dispersion at low frequencies even in dry rocks (this effect was only known in water-saturated samples) and they infer that it should result from remaining tightly bounded water present in very low amounts at the pores and difficult to remove. Thus, they conclude that some Maxwell-Wagner polarization occurs as well as, probably, some electrode polarization. Since then, the dielectric properties of unsaturated porous materials have attracted the effort of many researchers (e.g., Yale, 1985; Olhoeft, 1987), and at the end of the 1990s, a very complete review on this topic, presenting theoretical models and electric measurements, is done by Chelidze and Guéguen (1999) and Chelidze et al. (1999). More recently, a model combining low-frequency complex conductivity and high-frequency permittivity in unsaturated porous media is developed in a broad frequency range from $1 \mathrm{mHz}$ to $1 \mathrm{GHz}$ by Revil (2013). The basic concept describing the electric properties of unsaturated porous materials is the electrical double layer that coats the grains' surface and is formed by the Stern and diffusive layers (Revil, 2013). Charges in the double layer compensate the charges on the mineral surfaces and polarize this layer. This effect is named as $\alpha$ polarization caused by electromigration and charge accumulation; it is the dominant mechanism of polarization in a very broad frequency range (Revil, 2013). These models do not consider the case where only aggregates of bound water are formed (below the capillary regime). Even so, the interest in the transport process of confined water in porous materials has been growing recently.

\footnotetext{
Manuscript received by the Editor 26 October 2013; revised manuscript received 12 February 2014; published online 9 May 2014.

${ }^{1}$ University of Évora, Geophysics Centre of Évora, Évora, Portugal. E-mail: hgsilva@uevora.pt.

${ }^{2}$ University of Évora, Geophysics Centre of Évora and Physics Department, Évora, Portugal. E-mail: tlem@uevora.pt; mourad@uevora.pt; rrosa@uevora.pt.

${ }^{3}$ University of Aveiro, I3N-Physics Department, Aveiro, Portugal. E-mail: jmonteiro@ua.pt; mpfg@ua.pt; skm@ua.pt.

${ }^{4}$ University of Évora, Geophysics Centre of Évora and Geosciences Department, Évora, Portugal. E-mail: pmoita@uevora.pt.

${ }^{5}$ University of Évora, Geosciences Department, Évora, Portugal, and University of Aveiro, GeoBioTec Research Centre, Aveiro, Portugal. E-mail: apinho@ uevora.pt.

(C) 2014 Society of Exploration Geophysicists. All rights reserved.
} 
This is mainly due to the fact that porous materials can be used as contaminant reservoirs and neutron moderators in nuclear reactors (Hartl et al., 2012), among other applications.

Additionally, porous media are very important in the study of low-temperature behavior of water, especially the supercooled water phase transition (Brovchenko and Oleinikova, 2007). Water confined in the pores does not freeze below the bulk freezing point, $T_{f} \sim 273 \mathrm{~K}$; instead, it forms a supercooled water state that is constituted by a mixture of two liquid states, low-density and highdensity water. When the critical temperature $T_{c} \sim 200-220 \mathrm{~K}$ is reached, these states finally freeze to low-density and high-density amorphous ice, respectively (Holten and Anisimov, 2012). A review of supercooled water studies can be found in Debenedetti (2003). Typically, these experiments involve techniques such as nuclear magnetic resonance (Mallamace et al., 2006), quasielastic neutron scattering (Qvist et al., 2011), small angle X-ray diffraction (Huang et al., 2010), and others.

In this context, dielectric spectroscopy can be used as a complementary technique to measure water phase transitions because water dominates the dielectric response through induced polarization. In fact, in the case in which it is not possible to form a double layer, bound water aggregates are created and significant polarization is expected to occur (water is an electrically polar molecule). Actually, dielectric spectroscopy has already been used to evaluate ice content below the bulk freezing point in porous materials (Bittelli et al., 2004). Moreover, Gomes et al. (2006), studying organic semiconductors, prove that the electrical instability known to exist in these materials was caused by the presence of confined water in the organic layer. This was evidenced by the observation of an anomaly in the current versus temperature curve that was clear for higher water contents. The authors explain the anomaly as follows: Water confined in the organic layers does not crystallize at the bulk freezing point $T_{f} \sim 273 \mathrm{~K}$; instead, it forms a metastable liquid down to $T_{c} \sim 200-220 \mathrm{~K}$ that behaves electrically as a charge trap and causes the anomaly. Below $T_{c}$, water solidifies, the traps disappear, and the current-versus-temperature curve recovers its normal behavior. In the case of dielectric measurements, below $T_{f}$, water aggregates maintain a similar polarization as in the liquid state, but as the temperature decreases, polarizability also decreases until these aggregates completely freeze at $T_{c}$, forming ice and reducing its polarization appreciably; this is reflected in a change in the dielectric spectral response of the material. This reduction is similar to what happens at $T_{f}$ in water-saturated porous media (Bittelli et al., 2004). The low-temperature dielectric behavior of confined water depends on the way water is bounded to the pore walls, and for that reason it is an important technique to study (frequency-dependent) water attachment to rock structure. Such water attachment is crucial in many aspects of petrophysics such as rock alteration, fluid transport, heat transfer, and others. In this perspective, measurement of the electric behavior of the rocks at the supercooled water phase transition is a very potential method and demands a deeper study.

Keeping this in mind, the present study is focused on the dielectric behavior of three granitic rocks from Évora (south of Portugal) affected by pore water in the frequency range from $100 \mathrm{~Hz}$ to $1 \mathrm{MHz}$ and temperatures of 100-350 K. The findings are related with the rocks' petrographic nature (grain size, mineral content, connected porosity, and specific surface area). A change in the dielectric response of the samples is found at $T_{c} \sim 200-220 \mathrm{~K}$ and is attributed to the supercooled pore water phase transition.
The rock presenting most clearly that change of dielectric behavior has a very low connected porosity (water removal from the pores is very difficult) and is the one with lower alteration degree (evidenced by the geochemical analysis). In fact, an important application of this type of measurements is the evaluation of the rock alternation degree because the mentioned change in the dielectric behavior depends on it. Finally, a first attempt to relate the critical temperature of the supercooled phase transition and the measuring frequency is presented; we find that the activation energy (defined below) and $T_{c}$ follow a logarithmic frequency dependence characteristic of confined diffusion processes (Heitjans and Kärger, 2005). The activation energy and critical temperature are related with the water attachment to the rock; thus, the evaluation of these parameters for different rocks is a likely technique to evaluated such attachment.

\section{ROCKS CHARACTERIZATION}

\section{Petrography}

The analysis of the constituent minerals, the texture, and structure of the three rocks studied, was done by optical mineralogy using thin sections of the rocks. The samples are identified as YC (yellow color and coarse grains), RM (rose color and medium grains), and GM (gray color and medium grains). The micrographs of the three rocks studied here are shown in Figure 1.

The YC rock specimen exhibits a hypidiomorphic to allotriomorphic texture whose matrix has an average granularity of approximately 3-4 $\mathrm{mm}$. The porphyritic texture, more evident on the hand sample scale, results from the presence of feldspars with higher dimensions (phenocrysts $\sim 10-15 \mathrm{~mm}$ ) within a finer matrix. Additionally, the elongation of phenocrysts defines, on a hand specimen scale, a slight anisotropy (preferred orientation). YC (Figure 1a) has a light color, leucocratic, where the mafic minerals (ferromagnesium dark minerals) do not exceed $1 \%-2 \%$ of the total rock volume. The modal composition of the rock is feldspars $(60 \%-70 \%)$, quartz $(35 \%-40 \%)$, biotite $(1 \%-2 \%)$, and muscovite $(\sim 3 \%-4 \%)$. The accessory phases observed (very small amount) are apatite and zircon. Chlorite (also rare) was observed as a secondary phase substituting primary (igneous phases).

The RM rock sample (Figure 1b) exhibits predominantly an allotriomorphic to hypidiomorphic medium to fine-grained texture (1 to $2-3 \mathrm{~mm}$ ). The main mineralogy is feldspar $(65 \%-75 \%)$, quartz $(25 \%-35 \%)$, biotite $(1 \%-2 \%)$, and muscovite $(2 \%-3 \%)$. The accessory phases are opaque minerals $(<1 \%$ volume) and apatite. It is a light-colored rock where dark minerals do not exceed $1 \%-2 \%$ of total volume. Essentially, with the naked eye, the existence of quartz clusters stands out partially edged by biotite. These clusters, slightly elongated, reach 8-10 mm long in a finer matrix, which, underlined by biotite, gives the rock a preferred orientation look. Opaque minerals are rare $(<1 \%)$ and occur scattered through the rock forming euhedral to subhedral crystals that are larger than those associated with biotite. Apatite is a rare accessory phase that occurs together with felsic phases.

The GM sample (Figure 1c) has a hypidiomorphic texture with a granularity average around $2 \mathrm{~mm}$. The main paragenesis is feldspar $(65 \%-75 \%)$, biotite $(10 \%-15 \%)$, quartz $(30 \%-40 \%)$, and muscovite $(1 \%)$. As accessory phases, apatite, zircon, and a staurolite crystal were observed. The secondary phases are calcite and chlorite. 
In summary, we can state that the three studied rocks correspond to granitoid specimens, that is, granites s.l. Nevertheless, some differences can be observed that correspond to different classifications. The YC and RM samples have very similar mineralogical compositions (modal composition), classified as granite s.s., as well as a slightly preferred orientation (the minerals are weakly aligned with each other defining a planar anisotropy). The most significant differences between these rocks are the porphyritic texture (bigger crystals of feldspar stand from a thinner matrix) and the higher degree of alteration of sample YC. The GM sample is different because it has more plagioclase, less alkaline feldspar, and more biotite than YC and RM, categorized as a granodiorite. The main aspects are presented in Table 1.

Complementarily, we did scanning electronic microscopy (SEM) for the three rocks, but we will only discuss the results for GM, Figure 2.

\section{Geochemical analysis}

The geochemical analyses of the three studied specimens are presented in Table 2. They agree with the above petrographic description and classification; they roughly correspond to granitoids (or granites s.1.), but geochemically (e.g., in terms of major elements) they can be distinguished in granites s.s. (YC and RM) and granodiorite (GM). The GM is, relative to the others, less differentiated (less alkali and more iron-magnesium content) being compositionally closer to more basic rocks (e.g., diorite).

\section{Porosity measurements}

Porosity of igneous rock depends on the following factors: particle size distribution, shape of particles and orientation, and fracturing and alteration influences (Pinho, 2003). It can be defined by the ratio between the volume of voids and the total volume of the rock expressed as a percentage. Here, we determine the connected porosity $\phi_{a}$, in which the void volume measured corresponds only to the volume of the open interstices accessible to water. Their determination is made by the buoyancy method suggested by the International Society of Rock Mechanics (ISRM, 1979). YC has an apparent porosity of $\phi_{a}=1.62 \%$, which is slightly higher from the typical values for granites, $\sim 1.0 \%$, and it could correspond to some level of alteration of this rock. Second, RM has an apparent porosity of $\phi_{a}=1.39 \%$, which is still higher from the characteristic values for granites. Finally, GM has an apparent porosity of $\phi_{a}=1.10 \%$, which is a usual value for granites. These values appear in Table 3 and support the observation that the alteration degree increases from GM to RM and to YC.

\section{Specific surface area measurements}

Nitrogen adsorption-desorption isotherms and krypton adsorption data were determined, at $77 \mathrm{~K}$, on the Autosorb-iQ from Quantachrome Instruments. It used helium (for dead-space calibration) and nitrogen of $99.999 \%$ purity, supplied by Linde and Air Liquide, respectively; krypton ( $99.99 \%$ purity) was supplied by Linde. Prior to the adsorption measurements, the rock samples were cut into small pieces of similar size, ultrasonic cleaned with distilled water, dried at $373 \mathrm{~K}$, and outgassed for $8 \mathrm{~h}$ at $393 \mathrm{~K}$; this temperature was achieved using a heating rate of $1 \mathrm{~K} / \mathrm{min}$. The uptakes are expressed per gram of the outgassed material. The representative nitrogen adsorption-desorption isotherms shown in Figure 3 present a similar behavior discussed below. In all cases, the adsorption isotherms approach type II of the IUPAC classification (Sing et al., 1985), but the full adsorption-desorption isotherms exhibit, at high $\mathrm{p} / \mathrm{p}^{o}$, type H3 hysteresis loops (Sing et al., 1985; Rouquérol et al.,

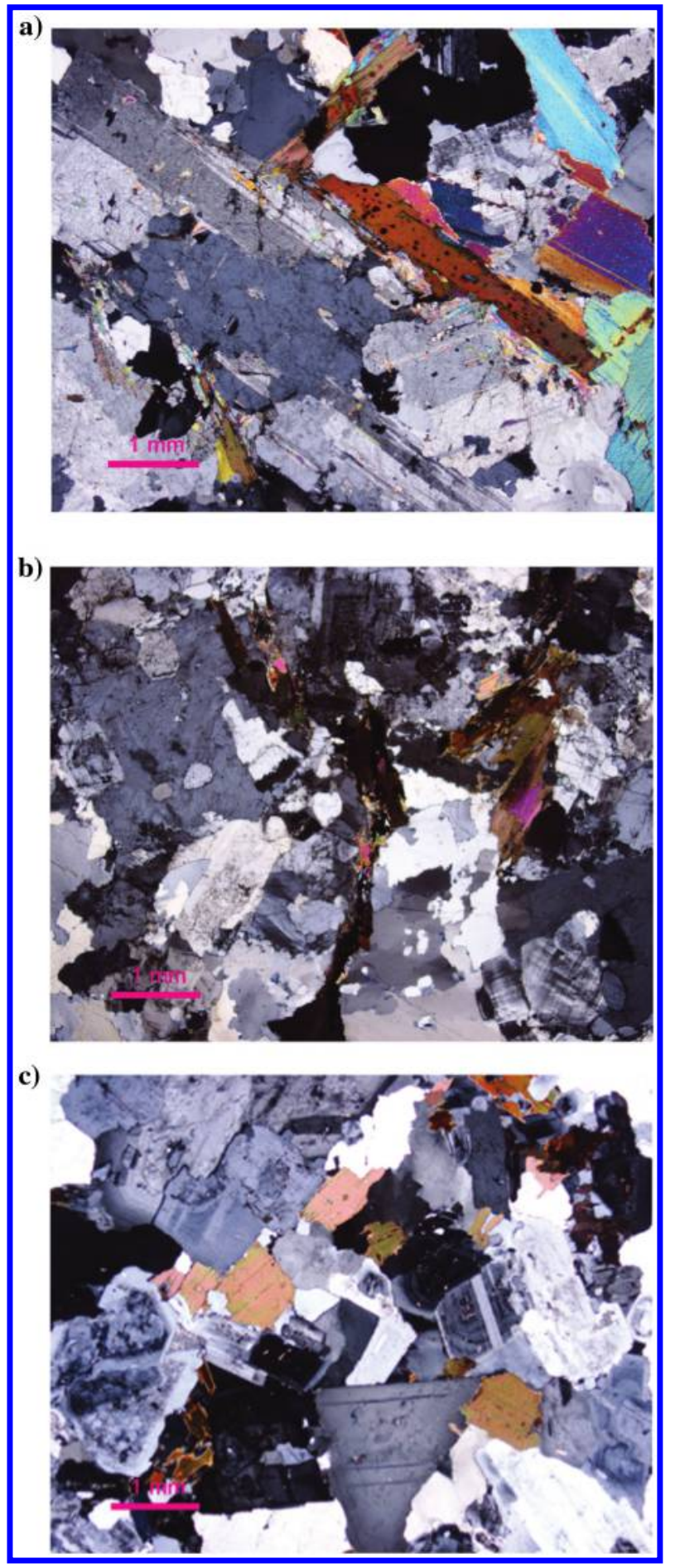

Figure 1. Micrographs of the three granitic rock types considered in this work: (a) YC (yellow color and coarse grains), (b) RM (rose color and medium grains), and (c) GM (gray color and medium grains). 
1999). This type of isotherm, now termed type IIb (Rouquérol et al., 1999), is generally obtained with aggregates of platelike particles giving rise to slit-shaped pores (Sing et al., 1985). The amount of adsorbed gas by the rock samples were in all cases very low but measurable, and small differences could be detected among them. From the analysis of nitrogen adsorption by the BrunauerEmmett-Teller (BET) method and considering $0.162 \mathrm{~nm}^{2}$ for the molecular cross-sectional area (Sing et al., 1985; Rouquérol et al., 1999), specific surface areas of $0.09,0.14$, and $0.13 \mathrm{~m}^{2} \mathrm{~g}^{-1}$ were obtained, respectively, for GM, RM, and YC. The adsorptiondesorption process occurring at the pores is revealed by a significant inflection of the specific surface area curve for $\mathrm{p} / \mathrm{p}^{o}<0.1$. Taking into account the very small specific surface areas, krypton adsorption (Sing et al., 1985) was also determined at $77 \mathrm{~K}$. Similar values and tendency were obtained from the application of the BET equation and considering $0.205 \mathrm{~nm}^{2}$ for the krypton cross-sectional area. Therefore, the results are statistically valid and appropriate for comparing the samples; see Table 3.

\section{DIELECTRIC MEASUREMENTS}

\section{Method}

Thin cylindrical samples with approximately $24 \mathrm{~mm}$ diameter and 2-4-mm thicknesses were prepared. Once cut and carefully polished (with a $15-\mu \mathrm{m}$ polishing disk), the samples were cleaned in an ultrasound bath and heated from room temperature (RT) up to $\sim 400 \mathrm{~K}$ and after that cooled down again to RT. Circular electrodes with a diameter of $20 \mathrm{~mm}$ were then established using $\mathrm{RS}^{\circledR}$ silver conductive paint (a 10 min heat cure was done to increase electrode conductivity as recommended by RS). Samples with different water contents were prepared as discussed below. They were then placed in the cryostat, and the temperature was lowered to $100 \mathrm{~K}$ at a rate of $\sim 1 \mathrm{~K} / \mathrm{min}$; after that, dielectric measurements were performed in $\sim 10 \mathrm{~K}$ steps up to $350 \mathrm{~K}$ for frequencies in the range from $100 \mathrm{~Hz}$ to $1 \mathrm{MHz}$. A liquid nitrogen bath cryostat (this system uses helium as the contact gas) was used, and an Oxford ITC4 controlled the temperature.

Two sets of measurements were done:

1) The samples (representative from the three rocks) were oven dried at $\sim 400 \mathrm{~K}$ over approximately $12 \mathrm{~h}$, removed from the oven, and passed to the cryostat to perform the measurements. This raises problems related to the fact that during the passage from the oven to the cryostat, the samples absorb water from the ambient causing the measurements to vary according to the environment conditions (temperature, relative humidity, etc.)
Nevertheless, it permits us to study the effect of small water contents in the samples (below the capillary regime).

2) The GM sample was measured, completely water saturated, and vacuum dried inside the cryostat. Saturation was achieved by immersing the sample in distilled water at $\sim 400 \mathrm{~K}$ for about $15 \mathrm{~min}$; the sample was then removed from the water bath (droplets blown out) and placed in the cryostat, and the temperature was lowered in the same way as the first set of measurements. Once finished with the dielectric spectroscopy measurements, the sample was left in the cryostat under primary vacuum for $12 \mathrm{~h}$ at $\sim 400 \mathrm{~K}$ and measurements repeated. This procedure (12 $\mathrm{h}$ of drying in vacuum and dielectric measurements) was repeated in the two following days, and it was verified that there is no appreciable difference in the measurements for drying periods above one day (consistent with the presence of tightly bound water in the pores, which is difficult to remove); thus, we will study only this case.

The impedance measurements were done using an Agilent 4294A precision impedance analyzer, in the frequency range between $100 \mathrm{~Hz}$ and $1 \mathrm{MHz}$, applying a $V_{\mathrm{AC}}=1 \mathrm{~V}$, and measuring in the $C_{p}-R_{p}$ configuration (capacitance and resistance in parallel). From the measured $C_{p}$ (capacitance) and $R_{p}$ (resistance) values, it is possible to calculate the real and imaginary part of the (relative) complex permittivity $\varepsilon^{*}=-j$ using equations 1 and 2 :

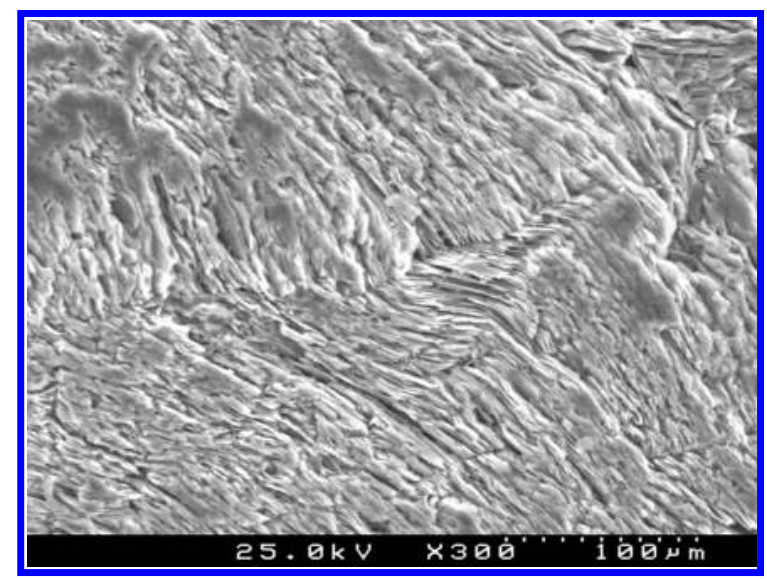

Figure 2. SEM photograph of a section of the GM rock.

Table 1. Characteristics and descriptive comparison of the three rock samples analyzed in section 3 and identified as YC (yellow color and coarse grains), RM (rose color and medium grains), and GM (gray color and medium grains).

\begin{tabular}{lllll} 
Rock & Name & \multicolumn{1}{c}{ Texture } & \multicolumn{1}{c}{ Ferromagnesian minerals } & Alteration \\
\hline YC & Granite s.s. & $\begin{array}{l}\text { Grain: 3-4 mm. Porphyritic and slightly } \\
\text { preferred orientation }\end{array}$ & Biotite $<1 \%-2 \%$ vol. & Residual (sericitization) \\
RM & Granite s.s. & $\begin{array}{l}\text { Grain: 1 to 2-3 mm. Slightly preferred } \\
\text { orientation and rare plastic deformation }\end{array}$ & $\begin{array}{l}\text { Biotite }<1 \%-2 \% \text { vol. Opaque } \\
\text { minerals (probably oxides) <1\% vol }\end{array}$ & Not significant \\
GM & Granodiorite & $\begin{array}{l}\text { Grain: 2 mm. Plastic deformation on quartz } \\
\text { (undulose extinction and subgranulation) }\end{array}$ & Biotite $\sim 10-15 \%$ & $\begin{array}{l}\text { Reduced (sericitization; calcite } \\
\text { growth, sauricitization) }\end{array}$ \\
\hline
\end{tabular}




$$
\begin{gathered}
\varepsilon^{\prime}=\frac{d}{A} \frac{C_{p}}{\varepsilon_{0}}, \\
\varepsilon^{\prime \prime}=\frac{d}{A} \frac{1}{\omega R_{P} \varepsilon_{0}},
\end{gathered}
$$

where $\omega$ is the angular frequency, $d$ is the sample thickness, $A$ is the electrode area, and $\varepsilon_{0}$ is the empty space permittivity. In the analysis of the dielectric data it is used the concept of activation energy $\left(E_{a}\right)$ used in semiconductor device characterization (Sze, 1981). This value is calculated from the Arrhenius equation, equation 3 , and it gives an indication of the thermal activation of the property that is being measured:

$$
F(T)=F^{(0)}+F^{(1)} \times \exp \left[-E_{a} /\left(k_{B} T\right)\right],
$$

where $F(T)$ can be the real or imaginary part of the dielectric permittivity, $F^{(0)}$ and $F^{(1)}$ are fitting constants, and $k_{B}$ is the Boltzmann constant.

It is important to mention that the rocks studied here are essentially isotropic at the macroscopic scale despite the small microanisotropies revealed by the thin sections. Furthermore, the sample-to-sample variation is found to be larger than the variation due to the measurement procedure and instrumental error; thus, the tensorial nature of the dielectric permittivity is inappreciable.

\section{First set of measurements (all samples)}

Figure 4 shows the dielectric constant $\varepsilon^{\prime}$ as a function of the frequency for several temperatures; it is visible that $\varepsilon^{\prime}$ increases from YC to RM, and mainly to GM for all the measuring frequencies. This could be related with the variation of biotite content in each rock (Saint-Amant and Strangway, 1970). Biotite is an iron-rich phyllosilicate mineral belonging to the mica group with a characteristic value $\varepsilon^{\prime} \sim 5-10$ (Saint-Amant, 1968), depending mostly on the chemical composition. Actually, GM has the highest percentage of biotite (10\%-15\%), decreasing significantly to RM and YC (less than $1 \%-2 \%$ ) as is summarized in Table 1 . Moreover, the dielectric constants of RM and YC have similar behavior, and the differences in values should be related with variations in their geochemical constituents; see Table 2.

In the low-frequency range $f \sim 100 \mathrm{~Hz}$, the contributions from the grain boundaries and sample-electrode interface polarizations are fundamental. In principle, grain boundaries are expected to be dominant in the case of dry rocks (Saint-Amant and Strangway, 1970). Naturally, the grain sizes determine the grain boundaries (smaller grains mean more boundaries), and this has a direct implication in the induced polarization at the grain boundaries. Indeed, grain size decreases from YC to RM and to GM followed by an increase of $\varepsilon^{\prime}$ (clearly seen in Figure 4). In particular, at $T=300 \mathrm{~K}$, the static $\varepsilon^{\prime}$ is approximately $\varepsilon_{s} \sim 6.5,9.5$, and 13.0, respectively, for YC, RM, and GM. This last rock has the lowest grain size, and therefore it is expected that GM presents the highest

Table 3. Connected porosity $\phi_{a}$ determined by the buoyancy method for the three rocks. Specific surface area (crosssectional area: $\sigma_{N 2}=0.162 \mathrm{~nm}^{2}$ and $\sigma_{\mathrm{Kr}}=0.205 \mathrm{~nm}^{2}$ ) is obtained from the BET method of nitrogen and krypton adsorption at $77 \mathrm{~K}$. Uncertainties are below the significant numbers of the values shown (a pessimistic evaluation was used).

\begin{tabular}{lccc}
\hline Rock & $\begin{array}{c}\text { Connected } \\
\text { porosity, } \phi_{a}\end{array}$ & $\begin{array}{c}A_{s}\left(\mathrm{BET}-N_{2}\right) \\
\mathrm{m}^{2} \mathrm{~g}^{-1}\end{array}$ & $\begin{array}{c}A_{s}(\mathrm{BET}-\mathrm{B}) \\
\mathrm{m}^{2} \mathrm{~g}^{-1}\end{array}$ \\
\hline $\mathrm{YC}$ & $1.62 \%$ & 0.13 & 0.12 \\
$\mathrm{RM}$ & $1.39 \%$ & 0.14 & 0.14 \\
$\mathrm{GM}$ & $1.10 \%$ & 0.09 & 0.06 \\
\hline
\end{tabular}

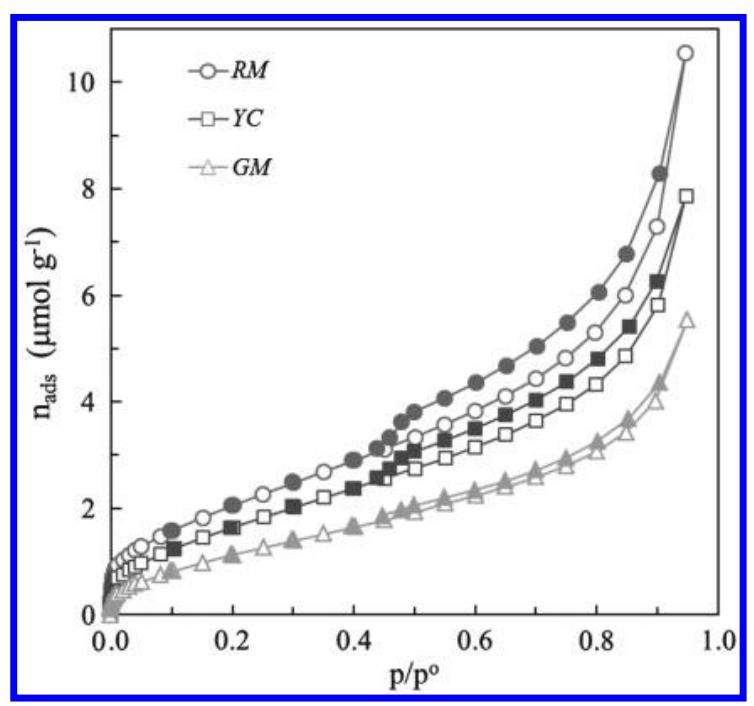

Figure 3. Nitrogen adsorption-desorption isotherms determined at $77 \mathrm{~K}$ of the three rocks (empty symbols, adsorption; filled symbols, desorption).

Table 2. Chemical composition, in terms of percentages of major elements (weight percent $>0.1 \%$ ) of studied samples. Here,

\begin{tabular}{|c|c|c|c|c|c|c|c|c|c|c|c|c|}
\hline Rock & $\mathrm{SiO}_{2}$ & $\mathrm{Al}_{2} \mathrm{O}_{3}$ & $\mathrm{Fe}_{2} \mathrm{O}_{3}^{(*)}$ & $\mathrm{MnO}$ & $\mathrm{MgO}$ & $\mathrm{CaO}$ & $\mathrm{Na}_{2} \mathrm{O}$ & $\mathrm{K}_{2} \mathrm{O}$ & $\mathrm{TiO}_{2}$ & $\mathrm{P}_{2} \mathrm{O}_{5}$ & LOI & Total \\
\hline YC & 73.81 & 14.39 & 1.21 & 0.03 & 0.19 & 0.58 & 3.34 & 4.81 & 0.116 & 0.39 & 0.82 & 99.69 \\
\hline GM & 71.02 & 15.51 & 2.57 & 0.051 & 0.96 & 3.07 & 4.25 & 2.54 & 0.383 & 0.12 & 0.42 & 100.9 \\
\hline
\end{tabular}
$\mathrm{Fe}_{2} \mathrm{O}_{3}{ }^{(*)}$ represents total iron $\left(\mathrm{Fe}^{2+}\right.$ and $\mathrm{Fe}^{3+}$ ); LOI - loss on ignition (all water content of the rock $\mathrm{H}_{2} \mathrm{O}^{+}$and $\mathrm{H}_{2} \mathrm{O}^{-}$). Uncertainties are below the significant numbers of the values shown (a pessimist evaluation was used). 


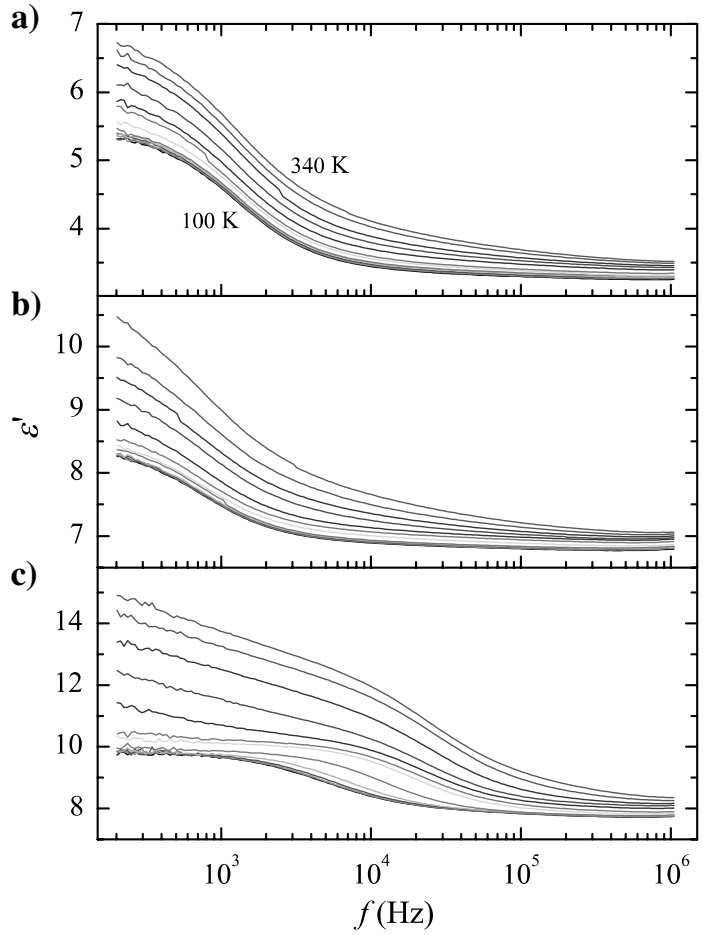

Figure 4. Real part of the permittivity, $\varepsilon^{\prime}$, as a function of frequency in the temperature range from $100 \mathrm{~K}$ to $340 \mathrm{~K}$ : (a) YC, (b) RM, and (c) GM.

a)

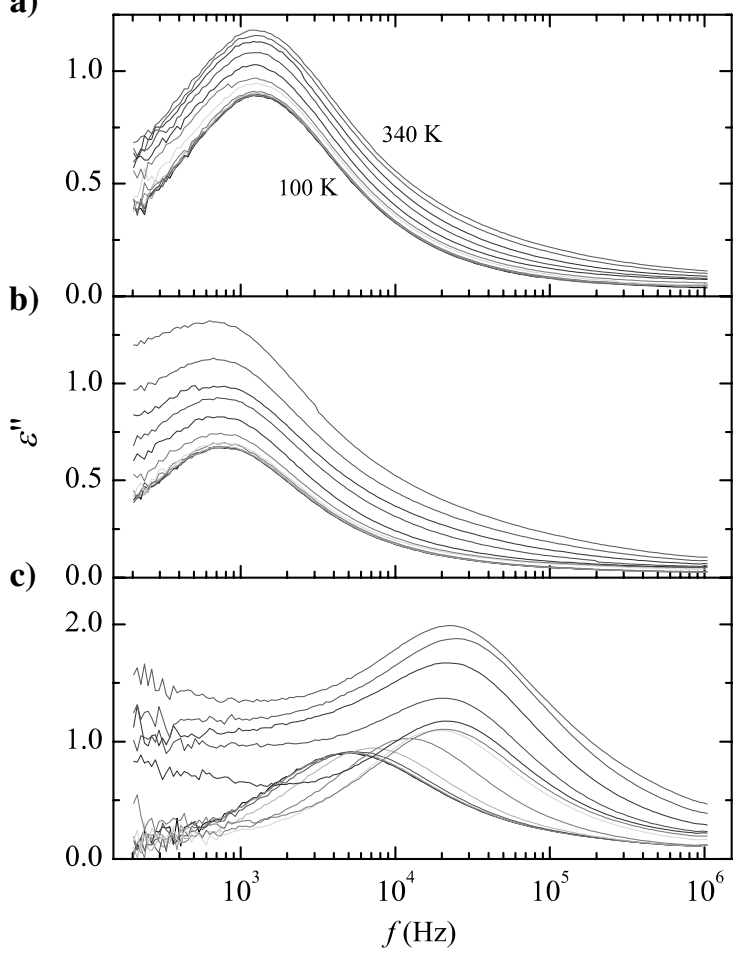

Figure 5. Imaginary part of the permittivity $\varepsilon^{\prime \prime}$ as a function of frequency in the temperature range from 100 to $340 \mathrm{~K}$ : (a) $\mathrm{YC}$, (b) RM, and (c) GM. permittivity at low frequencies. This result is also consistent with a decrease of the connected porosity from YC, RM, and GM, correspondingly. Moreover, in the three rocks, the $\varepsilon^{\prime}$ values increase with the temperature revealing a temperature-activated mechanism. The activation energies $E_{a}$ found for $f=200 \mathrm{~Hz}$ are on the order of $\sim 8.2,12.7$, and $10.2 \mathrm{~kJ} / \mathrm{mol}$ for YC, RM, and GM. The small $E_{a}$ values are not compatible with ionic charge transport (typical in water-saturated rocks). In the high-frequency range, 1-MHz limit, $\varepsilon^{\prime}$ tends in the three rocks to a constant value that is frequency independent and less thermally activated than the low-frequency limit. The typical values, at $T=300 \mathrm{~K}$, for the high-frequency dielectric constant are roughly $\varepsilon_{\infty} \sim 3.5,7.0$, and 8.0, respectively, for $\mathrm{YC}$, $\mathrm{RM}$, and GM. Also, the activation energies $(f=1 \mathrm{MHz})$ are on the order of 6.7, 6.0, and $8.2 \mathrm{~kJ} / \mathrm{mol}$ for YC, RM, and GM. Moreover, RM and GM have similar $\varepsilon_{\infty}$ values, significantly different from YC. Most importantly, the GM dielectric constant curves from 100 up to $220 \mathrm{~K}$ (the yellow curve in Figure 4) show an initial plateau followed by a dielectric relaxation typical of the MaxwellWagner polarization in inhomogeneous media. Nevertheless, above $220 \mathrm{~K}$, the dielectric constant curve changes to a relaxation behavior similar to RM and YC in which a significant dispersion appears for low frequencies. This temperature coincides with $T_{c}$ of supercooled water as reviewed previously (Debenedetti, 2003) and will be discussed below.

Furthermore, in Figure 5, the three samples show an evident dielectric relaxation dominated by one relaxation peak occurring at a given frequency. This relaxation frequency is practically temperature independent for YC and RM, $f_{\text {rel }} \sim 1 \mathrm{kHz}$. In fact, in the YC case, the frequency is slightly higher than in $\mathrm{RM}$ and it indicates that the dielectric dipoles relax easier in YC; see Figure 5a and 5b. On the contrary, the relaxation peak frequency in the GM case, Figure 5c, presents a different behavior because it changes with temperature; this evidences that a thermally activated process takes place. From 100 up to $180 \mathrm{~K}$, we found that $f_{\text {rel }} \sim 5 \mathrm{kHz}$; it increases up to $\sim 20 \mathrm{kHz}$ at $220 \mathrm{~K}$ and remains there (at least until $340 \mathrm{~K})$. Again, this transition coincides with the supercooled confined water transition and will receive more attention in the following sections. To clarify the previous findings, we represent $f_{\text {rel }}$ and $\varepsilon^{\prime \prime}\left(f_{\text {rel }}\right)$ as a function of temperature; see Figure 6. Two thermalactivated regimes are distinguishable in Figure $6 \mathrm{a}$ and $6 \mathrm{~b}$, one below $220 \mathrm{~K}$ (marked by the dashed vertical line) and other above this temperature. Actually, assuming an Arrhenius thermal dependence of the peak frequency $f_{\text {rel }}=f_{0}+f_{1} \exp \left[-E_{a} /(R T)\right]$, the activation energy is $\sim 13.8 \mathrm{~kJ} / \mathrm{mol}$ between 100 and $200 \mathrm{~K}$ (marked as a red curve) and $\sim 10.7 \mathrm{~kJ} / \mathrm{mol}$ from 220 up to $350 \mathrm{~K}$ (marked as a blue curve). The first value is similar to the activation energy of the rotational relaxation time of bulk water $E_{a} \sim 13.5 \mathrm{~kJ} / \mathrm{mol}$ (Yamada et al., 2011), and it is modified to a less thermally activated relaxation process at $220 \mathrm{~K}$. In fact, an inflection point is also found at $220 \mathrm{~K}$ in the thermal temperature of $\varepsilon^{\prime \prime}\left(f_{\text {rel }}\right)$; see Figure $6 \mathrm{~d}$. As mentioned above, this temperature is coincident with the critical temperature of the supercooled water phase transition (Debenedetti, 2003) and provides sufficient evidence for the existence of a dielectric process occurring at that temperature (further discussion will be presented). It is important to mention that this thermal behavior of the relaxation peak is not seen in YC and RM samples and only in GM; that is, the rock with a smaller specific surface area, significant texture (SEM imaging, Figure 2), and the lowest degree of alteration (petrography and geochemistry). 
Finally, the Cole-Cole plots (Figure 7) show that the three rocks are dominated by a ColeDavidson relaxation (Nigmatullin and Ryabov, 1997) proved by a characteristic $\alpha \sim 1.0$ when fitting the data with the Havriliak-Negami equation (Havriliak and Negami, 1967). An evident dispersion mechanism starts in GM above $T \sim 240 \mathrm{~K}$.

\section{Second set of measurements (GM)}

To clarify the role of water in GM dielectric measurements, a second set of measurements was done (described above).

In the water-saturated case, $\varepsilon^{\prime}$ (Figure $8 \mathrm{a}$ ) and $\varepsilon^{\prime \prime}$ (Figure 8c) show the expected peak at $\sim 273 \mathrm{~K}$ caused by the freezing point of bulk water, the first-order phase transition (Bittelli et al., 2004). But, in the capillary regime, the effect of water confinement in the pores is almost unappreciable and the low-temperature supercooled water phase transition is not noticeable.

In the vacuum-dried case, $\varepsilon^{\prime}$ (Figure $8 \mathrm{~b}$ ) and $\varepsilon^{\prime \prime}$ (Figure 8d) show a change of thermal behavior at $T_{c} \sim 220 \mathrm{~K}$; the temperature increase of these quantities becomes more significant above this temperature. In fact, the derivatives of all curves have a maximum around $T_{c}$ and the curves of $\varepsilon^{\prime \prime}(T)$ for $f \sim 10 \mathrm{kHz}, 100 \mathrm{kHz}$, and $1 \mathrm{MHz}$ show local maximum about this temperature. Again, this temperature coincides with the supercooled water phase transition and is a decisive evidence of the role that confined pore water has in the low-temperature dielectric behavior of the GM sample.

To further confirm this finding, we perform a thermal analysis of $\varepsilon^{\prime \prime}(1 \mathrm{kHz}, 10 \mathrm{kHz}, 100 \mathrm{kHz}, 1 \mathrm{MHz})$ as presented in Figure 9. We start by fitting the $\varepsilon^{\prime \prime}$ data to the Arrhenius law, as shown in Figure $9 \mathrm{a}$. The activation energies thus found are represented in Figure $9 \mathrm{~b}$ as a function of the measuring frequency. These follow a logarithmic frequency dependence of the type, $E_{a}=E_{0} \ln \left(f / f_{0}\right)$, with $E_{0}=0.83 \mathrm{~kJ} / \mathrm{mol}$ and $f_{0}=1.7 \mathrm{THz}$. This value of the characteristic frequency $f_{0}$ is inside the microwave/far-infrared region of the electromagnetic spectrum and coincides with the relaxation frequency of water (Rønne et al., 1997). Moreover, the logarithm increase of $E_{a}$ with the frequency is characteristic of confined diffusion processes, studied in condensed matter physics (Heitjans and Kärger, 2005). In the present case, it reflects a greater interaction of water with the pore walls for increasing rotation frequencies. This mechanism should dominate the dielectric response in the case where the electrical double layer is not formed, as expected for small aggregates of water nonuniformly distributed in the pores (Mancinelli et al., 2010).

Finally, to estimate the effect of the measuring frequency on the transition temperature $T_{c}$, we subtract the fitted Arrhenius curves $\varepsilon_{\text {fitted }}^{\prime \prime}$ to the measured values $\varepsilon_{\text {measured }}^{\prime \prime}$ and calculate the quantity: $\Delta=\varepsilon_{\text {measured }}^{\prime \prime}-\varepsilon_{\text {fitted }}^{\prime \prime}$. In Figure $9 \mathrm{c}$, it is noticeable that deviation from the Arrhenius law (given by $\Delta$ ) depends strongly with the frequency; decreasing from $1 \mathrm{MHz}$ down to $1 \mathrm{kHz}$. Likewise, it is seen that the temperature at which this deviation is maximum $\Delta_{\max }$ depends on frequency; thus, we define the critical temperature as b)

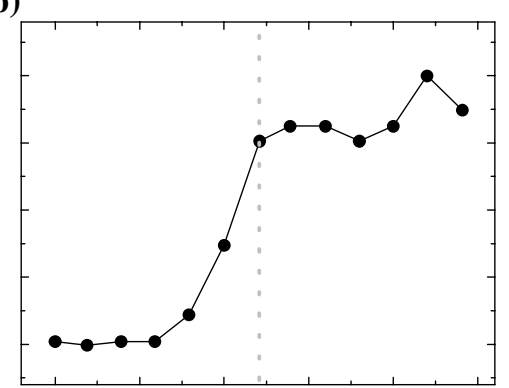

d)

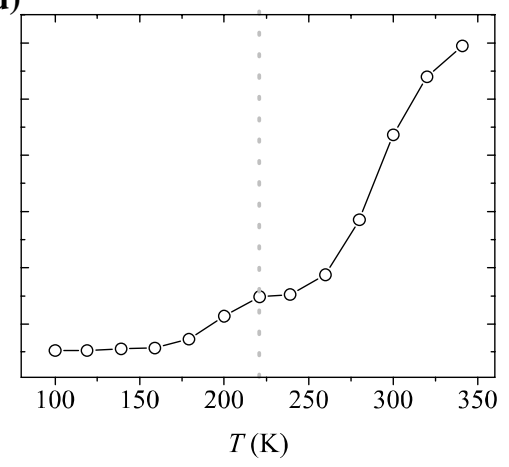

$(R T)^{-1}(\mathrm{~kJ} / \mathrm{mol})^{-1}$

helaxation peak frequency $f_{\text {rel }}$ and the imaginary part of the permittivity for that frequency $\left.\varepsilon^{\prime \prime}\left(f_{\text {rel }}\right)\right)$ of the GM sample. Panels (a and $\mathrm{b}$ ) represent $f_{\text {rel }}$ as a function of the inverse temperature and the temperature, respectively. Panels (c and d) show $\varepsilon^{\prime \prime}\left(f_{\text {rel }}\right)$ as a function of the inverse temperature and the temperature, respectively.

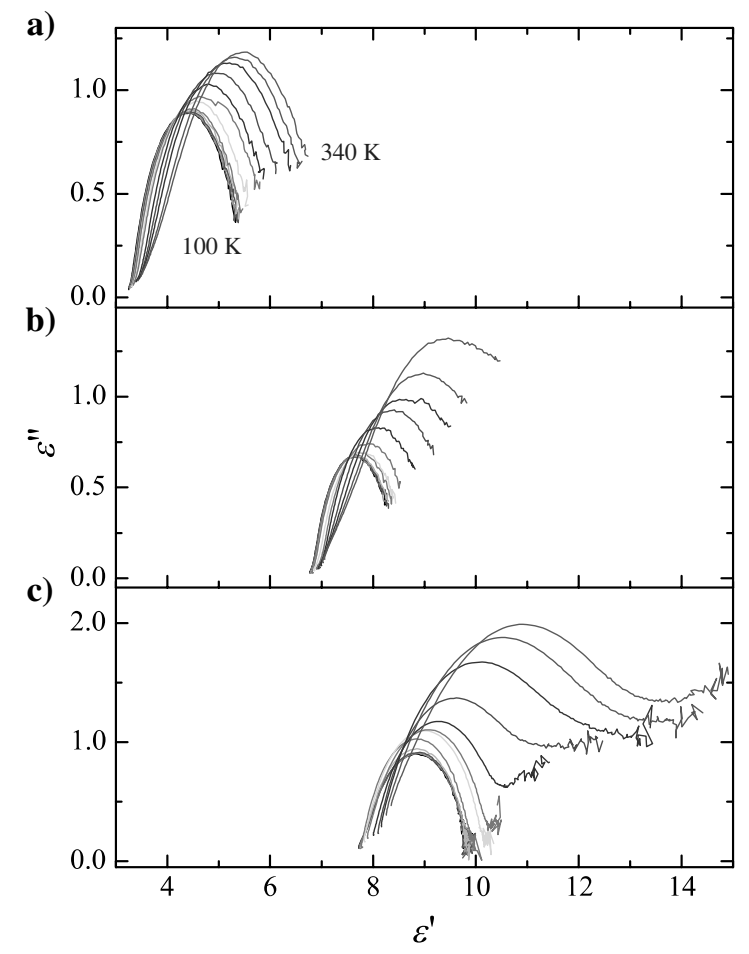

Figure 7. Cole-Cole plots for the three rocks considered: (a) $\mathrm{YC}$, (b) RM, and (c) GM. 
$T_{c}\left(\Delta_{\max }\right)$; see Figure $9 \mathrm{~d}$. It is observed that $T_{c}$ also depends logarithmically with frequency $T_{c}=T_{0}-T_{1} \ln \left(f / f_{0}\right)$, with $T_{0} \sim$ $317 \mathrm{~K}$ and $T_{1} \sim 5.6 \mathrm{~K}$.

\section{DISCUSSION}

The thermal dependence of the dielectric measurements of GM (first set of measurements, Figures 4 and 5) has a very distinct behavior over those of YC and RM; we argued that this is caused by confined (extrinsic) pore water. The main argument is the fact that $\varepsilon^{\prime}(f)$ and $\varepsilon^{\prime \prime}(f)$ curves of GM have different behavior below and above $T_{c} \sim 220 \mathrm{~K}$, and this is not seem in YC and RM; $T_{c}$ coincides with the supercooled water phase transition, and its effect in current versus temperature measurements was reported previously in organic semiconductors (Gomes et al., 2006). The mechanism causing the mentioned variation of the GM dielectric curves is the following: Below bulk water freezing point $T_{f}$, aggregates maintain a similar polarization as in the liquid state but decrease as temperature is reduced; when these aggregates completely freeze at the supercooled water phase transition $T_{c}$ to form ice, an appreciable reduction of its polarization occurs; this is reflected in a change of the dielectric spectral response of the sample.

Moreover, the geochemical analysis presented in Table 2 shows that YC has almost a double percentage of hydration (intrinsic) water as compared with RM and GM; nevertheless, GM is the sample that is mostly affected by the supercooled water phase transition. This fact should be an indication that pore (extrinsic) water is responsible for this effect. Actually, the geochemical analysis, the petrography, and the porosity reveal that the degree of alteration grows from YC to RM and to GM; rock alteration changes the way water interacts with its structure, in principle. A lower alteration degree should imply higher interaction.

Additionally, GM has a higher content of biotite compared with the other samples (Table 1); biotite has a significant affinity with water and could be responsible for the stronger interaction of the water molecules with the pore walls in GM. In accordance with this argument, GM presents a concentration of $\mathrm{CaO}$ almost three times higher than $\mathrm{YC}$ and $\mathrm{RM}$ (Table 2); $\mathrm{CaO}$ is one of the main agents of rock alteration that can easily bound water by a series of chemical process (Baltakys and Siauciunas, 2009) and could play a decisive role in the water interaction with the GM sample.

Also, GM has a smaller specific surface area when compared with YC and RM; when assuming a linear relation between cation exchange capacity and specific surface area (Woodruff and Revil, 2011), GM should have a lower cation exchange capacity than YC and RM.

Finally, the previous discussion is in agreement with the logarithmic frequency dependence of the activation energy (higher rotation frequencies become more difficult due the interaction of water molecules with pore walls) and the characteristic frequency found in the analysis $f_{0}=1.7 \mathrm{THz}$ is in the range of the typical frequencies of the relaxation response of water.

Figure 9. Analysis of the $\varepsilon^{\prime \prime}$ temperature behavior for a GM vacuum-dried sample. 


\section{CONCLUSIONS}

The results presented here point toward the conclusion that the $T \sim 220 \mathrm{~K}$ change in the dielectric measurements of the GM samples is associated with pore-water aggregates very difficult to be removed. This is consistent with the fact that YC and RM rocks have more structural water as indicated by geochemical analysis, but the effect is less noticeable compared to GM. Moreover, GM has lower porosity, alteration degree, and specific surface area of the three samples. The dependence of the low-temperature dielectric measurements on these three parameters is the main conclusion of the present work because it reveals a potential method to evaluate and study them. Actually, this result demands a theoretical understanding of the involved mechanisms, mainly because it will clarify rock alteration processes as they are rather important in applied petrophysics, e.g., monument conservation.

\section{ACKNOWLEDGMENTS}

One of the authors (H. G. S.) is grateful to the support from the Portuguese Science and Technology Foundation for the postdoc grant SFRH/BPD/63880/2009 and the Calouste Gulbenkian Foundation for the prize Estímulo à Criatividade e à Qualidade na Actividade de Investigaçãao (science program of 2010). The authors also acknowledge the support from the PEst-C/CTM/ LA0025/2011, RECI/FIS-NAN/0183/2012, and FCOMP-010124-FEDER-009331 PTDC/GEO-FIQ/4178/2012 projects. Moreover, we are thankful to the Portuguese companies from Évora, Anselmar, and Lusomarble, that provided us with the granitic rocks. Additionally, we are indebted to J. Figueira, S. Velez, and J. Velez for their crucial support in the sample preparation. Furthermore, we are grateful to M. R. Carrott and the Évora Chemistry Centre for kindly making the specific surface area measurements. A special acknowledgement is directed to H. L. Gomes (University of Algarve) because he introduced us to the topic of water phase transition. Finally, we are truly thankful to two anonymous reviewers and J. H. Schön for the crucial and rather constructive comments made to our manuscript.

\section{REFERENCES}

Baltakys, K., and R. Siauciunas, 2009, Physically and chemically bound $\mathrm{H}_{2} \mathrm{O}$ in the gyrolite structure: Materials Science Poland, 27, 255.

Bittelli, M., M. Flury, and K. Roth, 2004, Use of dielectric spectroscopy to estimate ice content in frozen porous media: Water Resources Research, 40, W04212, doi: 10.1029/2003WR002343.

Brovchenko, I., and A. Oleinikova, 2007, Effect of confinement on the liquid-liquid phase transition of supercooled water: Journal of Chemical Phvsics, 126, 214701, doi: 10.1063/1.2734963.

Chelidze, T. L., and Y. Guéguen, 1999, Electrical spectroscopy of porous rocks: A review - I. Theoretical models: Geophysical Journal International, 137, 1-15, doi: 10.1046/j.1365-246x.1999.00799x.

Chelidze, T. L., Y. Guéguen, and C. Ruffet, 1999, Electrical spectroscopy of porous rocks: A review - II. Experimental results and interpretation Geophysical Journal International, 137, 16-34, doi: 10.1046/j.1365246x.1999.00800.x

Debenedetti, P. G., 2003, Supercooled and glassy water: Journal of Physics: Condensed Matter, 15, R1669-R1726, doi: 10.1088/0953-8984/15/45 R01.

Gomes, H. L., P. Stallinga, M. Cölle, D. M. Leeuw, and F. Biscarini, 2006, Electrical instabilities in organic semiconductors caused by trapped super- cooled water: Applied Physics Letters, 88, 082101, doi: 10.1063/1 .2178410 .

Hartl, M., L. Daemen, and G. Muhrer, 2012, Water trapped in silica microspheres: Microporous and Mesoporous Materials, 161, 7-13, doi: 10 $.1016 /$ j.micromeso.2012.04.025.

Havriliak, S., and S. Negami, 1967, A complex plane representation of dielectric and mechanical relaxation processes in some polymers: Polymer, 8, 161-210, doi: 10.1016/0032-3861(67)90021-3.

Heitjans, P., and J. Kärger, eds., 2005, Diffusion in condensed matter: Methods, materials, models: Springer.

Holten, V., and M. A. Anisimov, 2012, Entropy-driven liquid-liquid separation in supercooled water: Scientific Reports (Nature), 2, 713, doi: 10 $.1038 /$ srep00713.

Howell, B. F., and P. H. Licastro, 1961, Dielectric behavior of rocks and minerals: American Mineralogist, 46, 269-288.

Huang, C., T. M. Weiss, D. Nordlund, K. T. Wikfeldt, L. G. M. Pettersson, and A. Nilsson, 2010, Increasing correlation length in bulk supercooled $\mathrm{H}_{2} \mathrm{O}, \mathrm{D}_{2} \mathrm{O}$, and $\mathrm{NaCl}$ solution determined from small angle $\mathrm{x}$-ray scattering: Journal of Chemical Physics, 133, 134504, doi: 10.1063/1.3495974.

ISRM, 1979, Suggested methods for determining water content, porosity, density, absorption and related properties and swelling and slake durability index properties: International Journal of Rock Mechanics and Mining Sciences \& Geomechanics Abstracts, 16, 141.

Mallamace, F., M. Broccio, C. Corsaro, A. Faraone, U. Wanderlingh, L. Liu, C.-Y. Mou, and S. H. Chen, 2006, The fragile-to-strong dynamic crossover transition in confined water: Nuclear magnetic resonance results: Journal of Chemical Physics, 124, 161102, doi: 10.1063/1.2193159.

Mancinelli, R., F. Bruni, and M. A. Ricci, 2010, Controversial evidence on the point of minimum density in deeply supercooled confined water: Journal of Physical Chemistry Letters, 1, 1277-1282, doi: 10.1021/jz100236j.

Nigmatullin, R. R., and Y. E. Ryabov, 1997, Cole-Davidson dielectric relaxation as a self-similar relaxation process: Physics of the Solid State 39, 87-90, doi: 10.1134/1.1129804.

Olhoeft, G. R., 1987, Electrical properties from $10^{-3}$ to $10^{+9} \mathrm{~Hz}$ : in Banavar, J. R., J. Klopik, and K. W. Winkler, eds., Proceedings of the 2nd International Symposium on Physics and Chemistry of Porous Media, AIP, 281-298.

Pinho, A. B., 2003, Geotechnical characterization of weak rock masses The Baixo Alentejo Flysch Group: Ph.D. thesis, University of Évora (in Portuguese).

Qvist, J., H. Schober, and B. Halle, 2011, Structural dynamics of supercooled water from quasielastic neutron scattering and molecular simulations: Journal of Chemical Physics, 134, 144508, doi: 10.1063/1 .3578472 .

Revil, A., 2013, Effective conductivity and permittivity of unsaturated porous materials in the frequency range $1 \mathrm{mHz}-1 \mathrm{GHz}$ : Water Resources Research, 49, 306-327, doi: 10.1029/2012WR012700.

Rønne, C., L. Thrane, P.-O. Åstrand, A. Wallqvist, K. V. Mikkelsen, and S. R. Keiding, 1997, Investigation of the temperature dependence of dielectric relaxation in liquid water by $\mathrm{THz}$ reflection spectroscopy and molecular dynamics simulation: Journal of Chemical Physics, 107, 5319, doi: 10 $.1063 / 1.474242$.

Rouquérol, F., J. Rouquérol, and K. S. W. Sing, 1999, Adsorption by powders and porous solids: Academic Press.

Saint-Amant, M., 1968, Frequency and temperature dependence of dielectric properties of some common rocks: M.S. thesis, Massachusetts Institute of Technology.

Saint-Amant, M., and D. W. Strangway, 1970, Dielectric properties of dry, geologic materials: Geophysics, 35, 624-645, doi: 10.1190/1.1440120.

Sing, K. S. W. D. H. Everett, R. A. W. Haul, L. Moscou, R. A. Pierotti, J. Rouquérol, and T. Siemieniewska, 1985, Reporting physisorption data for gas/solid systems with special reference to the determination of surface area and porosity (recommendations 1984): Pure and Applied Chemistry 57, 603-619, doi: 10.1351/pac198557040603.

Sze, S. M., 1981, Physics of semiconductor devices: Wiley Interscience.

Woodruff, W. F., and A. Revil, 2011, CEC-normalized clay-water sorption isotherm: Water Resources Research, 47, W11502, doi: 10.1029/ 2011WR010919.

Yale, D. P., 1985, Recent advances in rock physics: Geophysics, 50, 2480 2491, doi: $10.1190 / 1.1441879$.

Yamada, T., R. Yonamine, T. Yamada, H. Kitagawa, M. Tyagi, M. Nagao, and O. Yamamuro, 2011, Quasi-elastic neutron scattering studies on dynamics of water confined in nanoporous copper rubeanate hydrates: Journal of Physical Chemistry B, 115, 13563-13569, doi: 10.1021/ jp2029467. 